Disclosures B. Daou: None. K. Holste: None. G. Palmateer: None. B. Thompson: None. C. Lint: None. J. Elenbaas: None. C. Maher: None. A. Pandey: None.

\section{0-019 SAFETY AND EFFICACY OF CANGRELOR USE IN NEUROVASCULAR INTERVENTION: A MULTICENTER EXPERIENCE}

${ }^{1} \mathrm{G}$ Cortez, ${ }^{1} \mathrm{~A}$ Monteiro, ${ }^{2} \mathrm{~N}$ Sourour, ${ }^{2} \mathrm{~F}$ Clarençon, ${ }^{2} \mathrm{M}$ Elhoray, ${ }^{3} \mathrm{M}$ Grigoryan, ${ }^{3} \mathrm{~S}$ Mirza ${ }^{4} G$ Dabus, ${ }^{4}$ L Linfante, ${ }^{1} Y$ Murtaza, ${ }^{5} \mathrm{P}$ Aguilar-Salinas, ${ }^{1} \mathrm{~A}$ Aghaebrahim, ${ }^{1} \mathrm{E}$ Sauvageau, ${ }^{1} R$ Hanel*. ${ }^{1}$ Neurosurgery, Baptist Health System- Jacksonville, Jacksonville, FL; ${ }^{2}$ PitiéSalpêtrière Hospital, Paris, FRANCE; ${ }^{3}$ Adventist Health Glendale Comprehensive Stroke Center, Los Angeles, CA; ${ }^{4}$ Miami Neuroscience, Cardiac, and Vascular Institute, Baptist Hospital, Miami, FL; ${ }^{5}$ Neurosurgery, University of Arizona, Tucson, AZ

\subsection{6/neurintsurg-2020-SNIS.19}

Introduction/Purpose In patients undergoing acute neurointervention, thromboembolic complications are the most common procedure related morbidity, in special when endoluminal prosthesis (stents/flow diverters) are needed. Dual-antiplatelet therapy (DAPT) has been used in order to prevent these events. Cangrelor is a potent, intravenous, P2Y12-receptor antagonist presented as an alternative to oral antiplatelet agents, with a rapid onset and offset. We aimed to evaluate the safety and effectiveness of cangrelor in patients with acute neurologic ischemic disease and intracranial aneurysms that underwent endovascular intervention.

Materials and Methods We conducted a multicenter retrospective review of patients who underwent acute neurological intervention and had cangrelor as part of their optimal treatment. Patients from four comprehensive interventional neurovascular centers were included and allocated into two groups: (1) Acute ischemic (acute stroke and/or symptomatic atherosclerotic disease) and Aneurysms (ruptured and unruptured). Clinical presentation, laboratorial data, and outcomes were analyzed with an emphasis on periprocedural thromboembolic and hemorrhagic complications.

Results A total of 66 patients were included, 42 of whom were allocated in the ischemic group and 24 in the aneurysm group. In the acute ischemic group, median NIHSS was 15.5 (IQR, 8.25-21], and the majority of patients underwent mechanical thrombectomy (73\%) associated with either rescue intracranial stenting $(61.3 \%)$ or carotid stenting (35.4\%). The overall periprocedural complication in this group was $9.5 \%$, corresponding to $3(7.1 \%)$ postoperative symptomatic intracranial hemorrhages $(\mathrm{sICH})$ and $1(2.3 \%)$ intraoperative thromboembolic event. At discharge, a favorable outcome (mRS 0-2) was noted in $47.6 \%$, and $1(2.4 \%)$ patient died after the progression of a sizeable infarct. In the aneurysm group, $66.7 \%$ were ruptured, and the most common treatment modality was flow diverter (66.7\%). Although overall periprocedural complication was noted in 3 cases (12.5\%), only one complication (4.2\%) had occurred during or after cangrelor infusion. It consisted of a sICH in previously ruptured aneurysm. The other two complications occurred before cangrelor infusion, being 1 sICH and 1 thromboembolic event. At discharge, a favorable outcome (mRS 0-2) was seen in $66.7 \%$, and mortality was $8.3 \%$, related to the ruptured aneurysms that progressed with sICH. When both groups were divided according to cangrelor infusion duration, there was no significant difference in the number of complications in patients receiving either short (median of $2 \mathrm{~h}$ hours) or prolonged (median of 24 hours) infusion.

Conclusion Cangrelor is a safe alternative in patients requiring immediate intervention with the use of stents and flow diverters. Cangrelor was not associated with increased rates of hemorrhagic complications and also allowed the possibility for a secure transition to long-term DAPT and progression to surgery in the setting of unexpected complications. Ideally, prospective studies with larger samples and comparison with a standard antiplatelet regimen are required to clarify the best protocol, safety profile, and effectiveness of cangrelor in neurologic endovascular interventions, unlikely to happen on our field.

Disclosures G. Cortez: None. A. Monteiro: None. N. Sourour: None. F. Clarençon: None. M. Elhoray: None. M. Grigoryan: None. S. Mirza: None. G. Dabus: None. I. Linfante: None. Y. Murtaza: None. P. Aguilar-Salinas: None. A. Aghaebrahim: None. E. Sauvageau: None. R. Hanel: 1; C; Chiese USA, Inc. 2; C; Microvention, Codman, Stryker and Medtronic.

\section{0-020 ENDOLUMINAL BIOPSY FOR MOLECULAR CLASSIFICATION OF HUMAN BRAIN ARTERIOVENOUS MALFORMATIONS}

E Winkler*, D Wu, D McCoy, A Abla, D Cooke. University of California San Francisco, San Francisco, CA

\subsection{6/neurintsurg-2020-SNIS.20}

Introduction Brain arteriovenous malformations (bAVMs) are dysplastic tangles of blood vessels with direct arterial-venous shunting. Evidence has suggested that bAVMs may arise from different somatic or germ-line mutations. However, there are no methodologies to molecularly characterize bAVMs without open surgery. Whether different molecular subgroups may explain divergent natural histories remains unknown. We describe a novel endovascular biopsy (EB) technique to permit comprehensive genome-wide transcriptomic classification of bAVMs without need for open surgery in living patients.

Methods EB and computational fluid dynamic modeling (CFD) was performed during preoperative embolization. Cells were obtained from a bAVM juxta-nidal feeding artery and systemic iliac artery (control) with a platinum detachable coil and 0.035 -inch wire, respectively. Endothelial cells (ECs) were isolated with fluorescence assisted cell sorting (FACS) and sequenced on an Illumina HiSeq 4000 sequencer. Differential gene expression and gene ontology analyses were performed.

Results EB was successful in 4 patients without complication. FACS sorting detected viable ECs (CD31-positive, DAPI-negative) in each patient. EB yielded $269.0 \pm 79.9$ cells per biopsy (control: $309.2 \pm 86.6$ cells; bAVM: $228.8 \pm$ 133.4). Differential gene expression analysis demonstrated 106 differentially expressed genes in bAVM ECs (FDR < 0.05 ) (figure 1A), and clear separation of bAVMs and patient-matched controls on principal component and heatmap analyses (figure 1B). Differentially expressed genes were enriched for bAVM-related Ras-MAPK signaling and angiogenic cell motility pathways $(\mathrm{p}<0.05)$ (figure $1 \mathrm{C}$ ). When compared to open surgery, EB detected $83.3 \%$ of genes and genome-wide expression strongly correlated with ECs from open surgery $\left(\mathrm{R}^{2}=0.8\right)$. CFD modeling of blood 
flow, including wall shear stress and oscillatory shear index, correlated with EB gene expression and supported evidence of vascular remodeling.

Conclusions EB is a safe technique to permit non-invasive genome-wide sequencing of bAVMs. Transcriptomic data correlates well with tissues acquired with open surgery and implicates Ras-MAPK signaling in adult bAVMs. It is also now possible to correlate gene expression with CFD flow modeling in living patients. Further development of noninvasive diagnostic techniques to molecularly stratify patients are needed to better delineate divergent natural histories of bAVMs and potentially inform treatment planning.

Disclosures E. Winkler: None. D. Wu: None. D. McCoy: None. A. Abla: None. D. Cooke: None.
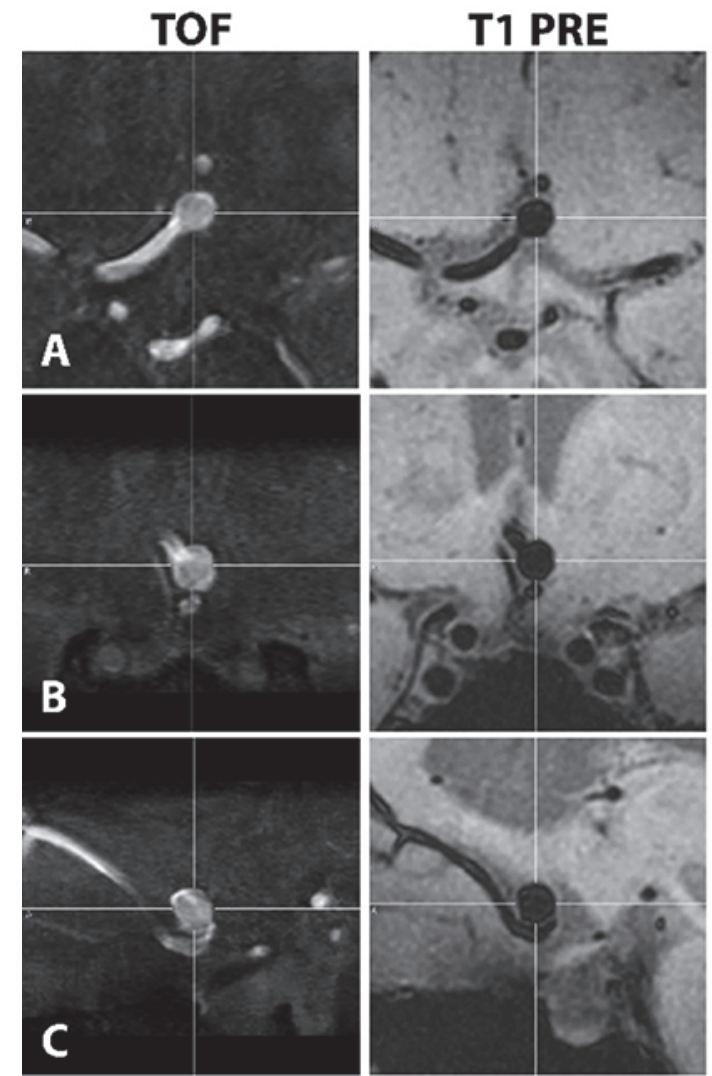

\section{0-021 INCREASED CONTRAST ENHANCEMENT OF THE PARENT VESSEL OF UNRUPTURED INTRACRANIAL ANEURYSMS IN 7T MR IMAGING}

${ }^{1}$ E Samaniego*, ${ }^{2} \mathrm{~J}$ Roa, ${ }^{3} \mathrm{H}$ Zhang, ${ }^{4} \mathrm{~T}$ Koscik, ${ }^{1} \mathrm{~S}$ Ortega-Gutierrez, ${ }^{5} \mathrm{G}$ Bathla, ${ }^{3} \mathrm{M}$ Sonka, ${ }^{5} \mathrm{C}$ Derdeyn, ${ }^{5} \mathrm{~V}$ Magnotta, ${ }^{6} \mathrm{D}$ Hasan. ${ }^{1}$ Neurology, Neurosurgery and Radiology, University of lowa Hospitals and Clinics, lowa City, IA; ${ }^{2}$ Neurology and Neurosurgery, University of lowa Hospitals and Clinics, lowa City, IA; ${ }^{3}$ Electrical and Computer Engineering, lowa Institute of Biomedical Imaging, University of lowa Hospitals and Clinics, lowa City, IA; ${ }^{4}$ sychiatry, University of lowa Hospitals and Clinics, lowa City, IA; ${ }^{5}$ Radiology, University of lowa Hospitals and Clinics, lowa City, IA; ${ }^{6}$ Neurosurgery, University of lowa Hospitals and Clinics, lowa City, IA

\subsection{6/neurintsurg-2020-SNIS.21}

Background Inflammation of the cerebral arterial wall may lead to aneurysm formation. The presence of aneurysm

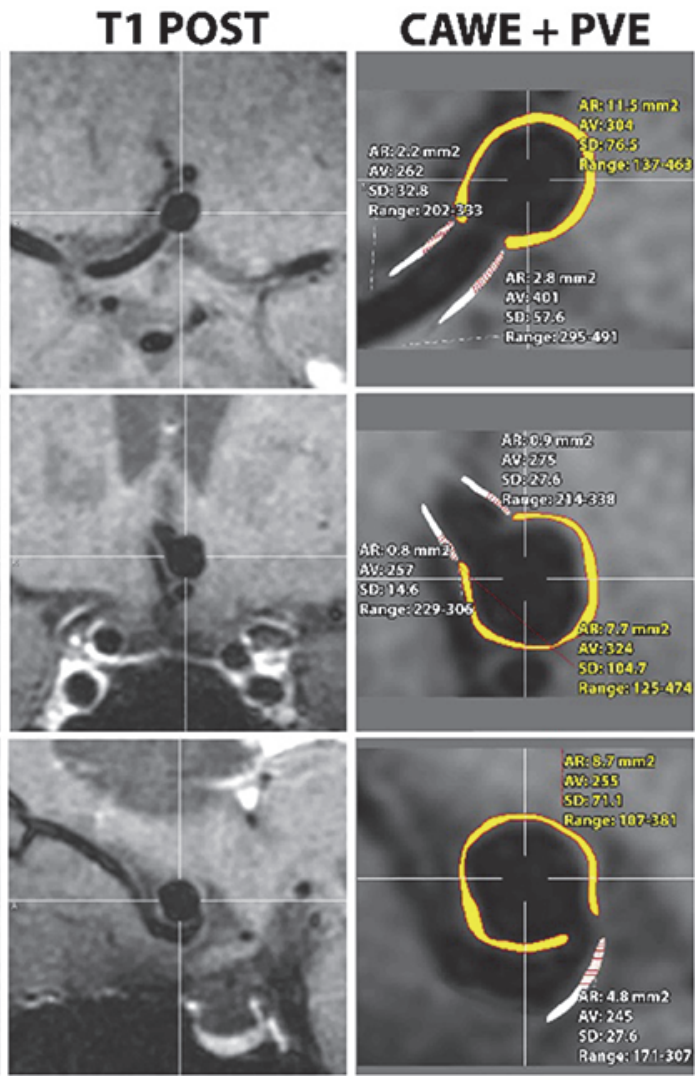

